\title{
De la diaspora noire : enseignements du contexte
} français

On the Black Diaspora: Lessons from the French Context

De la diáspora negra: enseñanzas del contexto francés

\section{Abdoulaye Gueye}

\section{(2) OpenEdition}

\section{Journals}

Édition électronique

URL : https://journals.openedition.org/remi/2710

DOI : 10.4000/remi. 2710

ISSN : $1777-5418$

Éditeur

Université de Poitiers

Édition imprimée

Date de publication : 1 mars 2006

Pagination : 11-33

ISBN : 2-911627-41-5

ISSN : 0765-0752

Référence électronique

Abdoulaye Gueye, «De la diaspora noire : enseignements du contexte français », Revue européenne des migrations internationales [En ligne], vol. 22 - $n^{\circ} 1$ | 2006, mis en ligne le 01 avril 2009, consulté le 15 avril 2022. URL : http://journals.openedition.org/remi/2710 ; DOI : https://doi.org/10.4000/remi.2710

Ce document a été généré automatiquement le 15 avril 2022.

(c) Université de Poitiers 


\title{
De la diaspora noire : enseignements du contexte français
}

\author{
On the Black Diaspora: Lessons from the French Context \\ De la diáspora negra: enseñanzas del contexto francés
}

Abdoulaye Gueye

1 Concept et objet d'étude, la diaspora noire est significativement présente dans la littérature anglo-américaine ${ }^{1}$. Déjà dans les années 1960, Shepperson en avait usé pour analyser le processus de dispersion du peuple noir et d'alliance de ses membres pardelà les frontières nationales (Edwards, 2001: 52). Les nombreuses conférences scientifiques, les programmes d'enseignement et les centres de recherche qui lui sont consacrés, et les débats passionnants suscités par Black Atlantic de Gilroy témoignent de son importance. Chivallon écrit que les sciences sociales françaises, par contre, ne manifestent pas un intérêt pour la diaspora noire (2004). Le présent article compte discuter ce constat. Il s'agira d'établir la qualité de réception de la diaspora noire dans la littérature et d'en rendre raison. Les questions posées sont les suivantes : pourquoi les sciences sociales françaises manquent-elles à s'ouvrir à cette réflexion? La diaspora noire, comme concept, est-elle inapte à éclairer les dynamiques, passées ou présentes, d'une certaine frange de la population de France? Je propose l'hypothèse principale suivante : le déficit de réflexion sur la diaspora noire renvoie à des facteurs structurels et institutionnels qui ont trait, d'une part, à la marginalisation en France d'une littérature à laquelle elle est historiquement et épistémologiquement associée, d'autre part, au rapport d'influence dans lequel s'inscrivent les sciences sociales françaises avec un État dont l'idéologie serait contredite par une promotion des études sur la diaspora noire, ainsi que dans la structuration même de ces sciences.

2 Selon mon hypothèse, la tâche à entreprendre est essentiellement d'ordre épistémologique. Elle consiste à dévoiler les fondements cachés d'une certaine portion de l'espace qu'est la recherche française, ainsi que les mécanismes par lesquels cet espace tend à se reproduire ou à changer, relativement à la diaspora noire (Bourdieu et Wacquant, 1992: 7). Pour ce faire, je puiserai dans l'armature théorique de Pierre Bourdieu qui offre avec le concept de champ un outil d'analyse opérant. Dans la 
définition complexe qu'il en donne, le champ renvoie d'abord à un espace de relations objectives entre des positions tenues par des agents et des institutions dotés de ressources spécifiques. Cet espace relativement autonome s'inscrit dans une relation plus ou moins inégalitaire avec d'autres champs dont notamment le champ du pouvoir, et fonctionne sur la base de règles ou règlements qui ne sont pas toujours codifiés ni explicites. Mais l'intérêt du concept de champ chez Bourdieu réside en ce qu'il met en lumière une part inconsciente dans le jeu des agents dont les actions ne relèvent pas toujours d'un choix rationnel mais aussi de dispositions inconsciemment intégrées, lesquelles découlent de leurs positions dans la structure où ils évoluent. Ainsi le point de vue des chercheurs ne suffit pas à lui seul pour comprendre le déficit de diaspora noire dans leur production. Il importe d'interroger l'histoire des sciences sociales, son développement et les luttes de positions qui s'y déroulent et dans le cadre desquelles opèrent les chercheurs. Pour d'autres raisons, le concept de champ est fort approprié ici. D'abord, il s'applique bien aux sciences sociales françaises qui sont une représentation, à une échelle réduite, du champ universitaire, dans la mesure où elles reproduisent la configuration hiérarchique, les règles, le fonctionnement et les enjeux de celui-ci. Les sciences sociales constituent, en effet, un réseau de compétition entre des positions qui revêtent parfois des caractéristiques méthodologiques, théoriques ou parfois idéologiques. Or, les théories et méthodologies différentes qui y ont bénéficié d'une certaine prédominance semblent manifester une incompatibilité épistémologique avec le développement de la réflexion sur la diaspora noire. Ensuite, bien qu'elles aient leurs propres règles et règlements, les sciences sociales ne sont pas indépendantes $d u$ champ politique d'où leur proviennent tantôt les objets de leurs propres interrogations tantôt leurs outils d'analyse. Cette relation n'est pas étrangère à leur besoin quasi structurel d'État, institution qui assure leur existence matérielle en tant que commanditaire et mécène.

3 Cet article est divisé en trois parties. La première est contextuelle ; elle démontre la validité du concept de diaspora noire et rend compte du processus sociohistorique de construction d'une diaspora noire en France. La deuxième partie, qui repose sur un recensement extensif de revues représentatives des sciences sociales ainsi que sur l'analyse de l'entrée de Black Atlantic dans l'édition française, établit l'absence de la diaspora noire dans cette production. La troisième partie, enfin, en identifie les causes.

\section{Concept et mise en contexte}

Le concept de diaspora noire procède de la juxtaposition d'un qualificatif racial - noir à un concept originellement forgé pour décrire l'expérience d'autres groupes que sont les peuples grec, d'abord, juif et arménien plus tard. Mais la différence d'historicités et d'expériences entre le peuple noir et les autres mentionnés le frappe-t-il d'incongruité ? Est-il inapproprié de parler de diaspora noire? Ces questions s'imposent au regard de la remarque de Schnapper, par exemple, sur la "véritable inflation » du strict concept de diaspora parfois utilisé à la place de termes plus appropriés selon elle : expatriés, exilés, réfugiés, etc. (2001:9).

Dans ses différentes acceptions, la réalité à laquelle renvoie le concept de diaspora a parfois différentes formes. C'est dire que le consensus est difficile à trouver dans le champ académique, ainsi que le montre la minoration par Clifford $(1994: 304)$ de ce qui constitue un critère essentiel de définition pour d'autres chercheurs, soit l'idée de 
retour à un foyer d'origine, réel ou imaginé, qui suppose, par déduction, le critère du territoire dont la signification est remise en question par Kastoryano (1992: 192) et Bordes-Benayoun (2000: 13). Parmi les chercheurs qui se sont le plus souciés d'avancer une définition rigoureuse, Safran (1991) décline des critères, largement repris par Cohen (1997: 26), qui aident à penser la diaspora noire: le déplacement, parfois traumatisant, d'un foyer originel ; la dispersion sur plus d'un territoire ; la formation d'une mémoire collective ; l'expérience d'une exclusion sociale fondée sur sa différence en tant que minorité ; et enfin le projet de retour au foyer d'origine. L'intérêt de cette définition est de suggérer que la diaspora est à la fois construction et essence. L'importance que Safran accorde à la mémoire collective par juxtaposition à l'origine territoriale en atteste. La mémoire collective, comme on le sait, est un socle à bâtir pour donner corps à un groupe; elle n'est donc pas donnée, elle est construite, dans la mesure où elle procède par sélection et rejet d'évènements, de symboles, d'institutions, de lieux (Halbwachs, 1968). De cette mémoire découlent, pour partie, une identité collective, qu'elle soit diasporique ou nationale, ainsi que le sentiment d'être solidaire, de former une communauté de destin pour un groupe d'individus différents tant par leur classe sociale, leur sexe, leur âge, leur religion... Il n'existe pas de diaspora sans cet effort collectif de se constituer comme telle par la mobilisation d'un passé, vrai ou fictif, et de symboles.

6 Comprendre ainsi la diaspora permet d'établir que, à partir du contexte géographique français, le concept de diaspora noire ne relève pas d'un usage inapproprié. Avec quelque retard sur les États-Unis, la France a été, en effet, un espace d'élaboration d'une diaspora noire. Au sortir de la Première Guerre mondiale, des contingents d'Africains y étudiaient, côtoyant quelques-uns de leurs compatriotes démobilisés de guerre dont le plus célèbre est le Sénégalais Lamine Senghor coopté au sein du Parti communiste français avant de prendre ses distances vis-à-vis d'une organisation dont le message trop prolétarien lui semblait inapte à exprimer la spécificité de l'exploitation dont étaient victimes les Noirs. Des colonies antillaises étaient venus environ 10000 travailleurs et étudiants issus de la petite bourgeoisie noire. À ces ressortissants des colonies françaises s'étaient ajoutés des intellectuels et artistes des États-Unis ou des Antilles britanniques (dont les célèbres Langston Hughes et Claude McKay) et des soldats noirs qui avaient décidé de ne pas regagner les États-Unis (Edwards, $2003: 3$ ). Cette présence noire ira grandissant au sortir de la Seconde Guerre : en 1960, l'Université française accueillait 4919 noirs africains (Ndiaye, 1962:60) qui avaient profité de l'élargissement de la nationalité française dans les colonies et de l'absence d'un cycle d'enseignement supérieur et parfois secondaire dans ces colonies. À la même période s'établissait une nouvelle génération d'écrivains américains déjà renommés dont Richard Wright et James Baldwin qui décèderont tous deux en France.

7 Ces flux traduisent le premier critère de formation d'une diaspora: le déplacement. Mais la distinction qu'établit Cohen (1997) entre déplacement forcé et déplacement volontaire pose la question de sa qualification. Une telle distinction ne s'avère pas forcément opérante, me semble-t-il, car la frontière entre ces deux types de déplacement n'est pas toujours identifiable. Le déplacement forcé serait-il à définir par rapport à l'usage de la violence contre un groupe par un autre groupe? La violence, forme de contrainte, peut-être aussi bien physique que symbolique soutient Bourdieu. La plupart des Noirs établis en France n'étant pas toujours expulsés de leur pays de naissance, on serait enclin à en déduire que leur déplacement est volontaire. Comment interpréter, cependant, le départ des Noirs américains confrontés au Jim Crow, à la 
ségrégation, celui des sujets coloniaux de la France dont l'hypothétique promotion socioprofessionnelle était conditionnelle à un niveau de scolarisation qu'il était impossible d'atteindre dans les colonies par manque d'infrastructures?

8 La germination d'une diaspora noire sur le sol français trouve son fondement dans ce déplacement. Celle-ci fut longtemps l'œuvre d'une élite intellectuelle qui en assura la responsabilité à travers la création de revues : ainsi, dans l'entre-deux-guerres, La voix des Nègres née en 1927, La Race nègre qui succéda à la précédente la même année, La dépêche africaine lancée en 1928 et L'Étudiant noir créé en 1934, puis, au sortir de la guerre, Présence africaine fondée en 1947.

9 À travers leurs noms, d'emblée, ces revues annoncent une conception transfrontalière de l'entreprise qu'elles sous-tendent. Elles entendent, en effet, produire à l'existence non pas un groupe défini par sa nationalité ou son territoire d'établissement, mais une communauté biologique et historique. D'où le recours systématique aux qualificatifs "nègre ", "noir " et "africain ». Ces qualificatifs expriment le déni délibéré des particularités nationales ou ethniques susceptibles de diviser pour mettre l'accent sur une communauté de destin fondée sur une histoire et une qualité biologique : l'identité de noir ou nègre. Les sous-titres des revues viennent d'ailleurs réaffirmer cet objectif : " organe de défense du comité de défense de la race nègre " pour La voix des Nègres; «revue culturelle du monde noir» pour Présence africaine, et "grand organe républicain indépendant de correspondance entre les Noirs ", pour La dépêche africaine. Sachant que le terme "Africain » était utilisé au sein de ce groupe comme synonyme de "noir » et "nègre ", mais souvent pour mettre en évidence les dimensions biologique et culturelle de la population ainsi désignée, l'ajout des épithètes "noir » et "nègre » aurait alors pour but d'éviter que les revues soient réduites à des entreprises initiées par et au service des ressortissants d'Afrique exclusivement. Il faut mentionner que ces revues étaient fondées, pour la plupart, par des natifs de l'Afrique.

10 En plus de leurs titres, les revues exprimaient leur objectif de production d'une communauté de destin noire à travers leur politique éditoriale. Ainsi, bien qu'elles fussent gérées en France et par des francophones, bon nombre d'entre elles publiaient une édition en langue anglaise. Un bilinguisme destiné à abattre l'obstacle, que constitue la différence linguistique, à la solidarité entre Noirs en facilitant la circulation des idées entre ceux des Amériques, de l'Europe et des colonies (Edwards, 2003).

11 Les articles constituaient, en dernière instance, les lieux d'énonciation de cet objectif. Dans son premier numéro, L'Étudiant noir, par exemple, déclarait ouvertement œuvrer pour « la fin de la tribalisation, du système clanique en vigueur au quartier latin » et la disparition de l'étudiant martiniquais ou sénégalais ou guadeloupéen au profit d'un "seul et même étudiant noir", en mobilisant, pour ce faire, non sur le critère de « l'anticolonialisme mais [de] l'appartenance raciale » (Blélard, $1981: 29$ ).

12 La prééminence accordée par cette revue au second critère contre le premier s'explique par deux raisons : a) l'expérience d'un groupe antérieur, Légitime défense, dont le projet d'unification des Noirs dans un front anticolonialiste s'était soldé par un échec à cause d'une inféodation au communisme qui en avait détourné bien des adhérents potentiels ; b) l'inaptitude de l'anticolonialisme à servir de liant à tout le peuple noir, puisque l'expérience coloniale ne peut objectivement pas s'appliquer qu'aux Noirs ni à l'ensemble des Noirs. 
13 La hiérarchie établie entre les deux critères participe de l'effort d'établissement des frontières d'une communauté noire. On sait, depuis Barth, cet effort consubstantiel à la formation de tout groupe, même diasporique (Barth, 1995). La comparaison de l'ensemble de ces revues montre, sur ce point, une convergence entre elles: ces frontières sont par elles données comme procédant de la juxtaposition d'une qualité biologique et d'une condition historique spécifiques. Concrètement, la diaspora noire y fait référence à une population dépositaire d'un patrimoine génétique qui atteste de son origine africaine et d'une mémoire collective fondée sur son expérience de la traite esclavagiste et de l'exploitation. Cette diaspora ainsi conçue, les revues en question en sont arrivées à faire du peuple noir une nation parfois enfermée dans des nations officiellement reconnues. Cet extrait de La Voix des Nègres est exemplaire :

[Q]uelques journaux français ont voulu, le 1er novembre dernier (le lendemain de notre assemblée générale), à tout prix, donner des renseignements (à leur façon) à leurs lecteurs sur ce qu'ils croyaient être notre comité. L'Écho de Paris s'est fait plus particulièrement remarquer; ce journal nous gratifie d'un esprit de pur nationalisme français en publiant « Les nègres sont des Français et veulent servir la France » (?). Or rien n'est aussi faux que cette affirmation! Les nègres ne sont d'aucune nationalité européenne et ne veulent servir les intérêts d'aucun impérialisme contre ceux d'un autre (Reproduit dans Edwards, 2003 : 31).

Dans cet extrait on relève le refus de laisser définir les «nègres » comme des Français ou des Européens. Ceci est un élément important d'analyse au regard des théories classiques qui ont montré que la référence à un pays, une patrie originelle à libérer ou à regagner un jour est un critère déterminant de définition de la diaspora (Safran, 1991 ; Cohen, 1997). Pour La Voix des nègres, les "nègres" n'ont pas à se fondre dans une nation française ou européenne, mais sont voués à réinvestir une patrie nègre momentanément sous l'occupation de puissances européennes. D'où "la ferme volonté » des "jeunesses nègres ", à travers cette revue, de lutter " pour la libération totale et l'émancipation de la race nègre » (cité dans Edwards, $2003: 31$ ).

Dans sa formation complexe, la diaspora noire a été commandée par l'inégalité et la discrimination dont les Noirs ont été victimes et auxquelles ils croient ne jamais pouvoir échapper hors d'un État nègre indépendant. Cette croyance est informée par les politiques et les pratiques imposées aux Noirs durant toute la première moitié du $\mathrm{xx}^{\mathrm{e}}$ siècle dans leurs différents pays d'installation. Marable montre que, aux États-Unis, à l'esclavage s'est substitué non pas la "démocratie multiculturelle » et «l'égalité raciale » tant promises au sortir de la guerre de Sécession, mais un système officiel de ségrégation privant les Noirs de la plupart des droits et avantages dévolus aux citoyens américains : les droits civiques leur seront tardivement concédés (1991). Au sein de l'Empire français, la distinction, longtemps maintenue, entre sujets et citoyens avait exclu l'écrasante majorité des Noirs d'une participation active à la vie économique et sociale. L'élargissement, après-guerre, de la nationalité française aux sujets coloniaux n'aura pas entraîné leur promotion au statut de citoyen à part entière, puisqu'il leur était refusé des droits et avantages dont jouissaient les citoyens français d'ascendance européenne. Présentant un article de loi, adopté en 1956, qui stipule que les étudiants français « dont le père est décédé alors qu'il était encore au service de l'AOF après y avoir servi pendant cinq ans au moins, pourront bénéficier d'allocations scolaires [...] pour la durée de toutes leurs études si celles-ci sont poursuivies en AOF [ou] dans les cas où les études sont effectuées en dehors de l'AOF, pendant un délai de deux années scolaires", Tévoedjre, un étudiant béninois, informé que ni les enfants des 
fonctionnaires africains en service en AOF ni ceux des fonctionnaires africains exerçant en métropole ne bénéficient de ces avantages, commentait, sarcastique :

«Le gouvernement français de la "Métropole" accepterait-il par exemple de CONTRIBUER ipso facto aux frais d'études des enfants d'un fonctionnaire africain décédé alors qu'il était en service en France... même depuis vingt-cinq ans ?

Ah! si j'avais la peau blanche, je me dépêcherais d'aller faire carrière au pays des Nègres...

... en attendant que le terrorisme y éclate » $(1956: 1-2)$.

Cette dialectique d'inclusion théorique et de discrimination formelle que souligne l'extrait précédent rapproche l'histoire du Noir de celle de la folie en société, c'est-àdire l'histoire de «l'Autre [...] à la fois intérieur et étranger, donc à exclure (pour en conjurer le péril intérieur) mais en l'enfermant (pour en réduire l'altérité) » (Foucault, 2003 : 15). D'elle, s'est aussi nourrie la construction d'une diaspora noire tout au long des années 1920 à 1950. Noirs d'Afrique, des États-Unis et des Antilles réunis en France convergeaient ainsi autour de l'idée qu'ils étaient soumis à un même destin déterminé par leur origine et leur qualité biologique.

17 La diaspora étant un processus qui se fait et se défait, ainsi que le font remarquer Patterson et Kelly (2000), la diaspora noire a connu un délitement à une certaine époque. Par contraintes d'espace et d'objectif je ne m'étendrai pas sur ce délitement, sauf pour mentionner qu'il est devenu visible entre le milieu des années 1960 et la première moitié des années 1980 , et a résulté, entre autres facteurs, de l'essoufflement de la dernière institution chargée de la fabrique de la diaspora noire, Présence africaine, de l'inexistence d'institutions de relève, du retour des intellectuels africains dans une Afrique indépendante pleine de promesses d'un avenir meilleur pour eux, de l'espoir suscité par le mouvement des droits civiques au sein de la population noire américaine, etc. Néanmoins, une dynamique de régénération de la diaspora noire est observable depuis la fin des années 1980. Elle s'inscrit dans un contexte d'accroissement de la présence en métropole d'une population antillaise estimée à plus d'un million et de renforcement d'une immigration africaine dont la sédentarisation

- impulsée par la législation restrictive sur l'immigration depuis 1974 - s'avère incontestable au regard de la cohabitation de trois générations (enfants, parents et grands-parents) sur le sol français. Les mêmes contraintes d'espace et d'objectif m'empêchent d'explorer ici les modalités et mécanismes de reconstruction de cette diaspora noire. Je relèverai néanmoins qu'ils sont élaborés au sein de nouvelles organisations au profil original, par comparaison à celles des années 1920-1950, puisqu'initiés et composés à la fois de natifs de la France métropolitaine, de naturalisés ou citoyens français nés en Afrique, aux Antilles ou ailleurs, ainsi que de simples résidents au sens de la Loi sur l'immigration. Parmi ces organisations figure l'Alliance des Personnes d'Ascendance Africaine en Europe (APAA) née à l'occasion du Forum Social Mondial de Seine Saint-Denis. Basée en France, elle compte aussi des représentations dans la plupart des capitales européennes, et plus significativement dans les pays dont la participation active à la traite négrière est aujourd'hui établie par les travaux d'historiens : Angleterre et Pays-Bas notamment. Déclinant la raison d'être de son organisation à l'occasion d'un entretien qu'il m'avait accordé en juillet 2005, l'un de ses fondateurs lui assignait une tâche similaire à celle dont des précurseurs de la diaspora noire tels W.E.B DuBois avaient investi leur mouvement, soit « la coordination politique et culturelle des intérêts des populations d'ascendance africaine à travers le monde » : 
Nous sommes en train de rassembler toutes les forces de la diaspora en vue de soutenir et aider les personnes d'ascendance africaine. [...] Notre but, ici sur le territoire européen, est de faire en sorte que dans une Europe qui se concrétise et où les décisions se prennent au plan multilatéral, les fils d'Afrique qui se trouvent sur ce continent se renforcent comme société civile responsable pour défendre les causes des descendants d'Afrique partout dans le monde. Nous travaillons pour la loi Taubira reconnaissant l'esclavage comme un crime contre l'humanité... nous travaillons avec des partenaires pour que cette loi soit reconnue partout en Europe et ailleurs. Nous allons renforcer notre volet lutte contre les discriminations. C'est nous qui avons lancé les directives pour la lutte contre les discriminations, dans le cadre de beaucoup de nos rencontres. (Edwards, 2001 : 46. Ma traduction)

À « l'appartenance raciale » qui avait servi à L'Étudiant noir de critère de délimitation des frontières du groupe qu'il voulait constituer, cette organisation offre, à travers les propos précédents, un équivalent, "l'ascendance africaine", juxtaposé dans ce discours aussi à la mémoire de la traite atlantique. Qui plus est, l'APAA développe à son tour l'idée d'un destin invariable et a-spatial des Noirs. Destin qui tiendrait à la discrimination contre laquelle les Noirs se heurteraient où qu'ils vivent, et justifierait qu'ils se regroupent pour « défendre [leurs] causes». On ne peut pas s'empêcher de se demander à quel point les peines prononcées ces dernières années par les tribunaux français pour discrimination raciale ainsi que les preuves de discrimination établies par quelques organisations antiracistes contre des individus et des entreprises ont nourri les actions de l'APAA.

19 La présente section a permis d'établir que la France a constitué un lieu d'élaboration et de manifestation d'une diaspora noire et d'éprouver la validité de ce concept. Il sera question, dans la section prochaine, de démontrer le déficit de réflexion sur la diaspora noire dans les sciences sociales françaises.

\section{État des lieux}

L'intérêt porté à un concept ou un objet d'études se mesure à quelques signes. Le lancement d'une revue ou la parution de plusieurs numéros de revues importantes lui étant spécialement consacrés en constituent des thermomètres fiables. L'ethnicité, le genre et le développement sont des exemples d'objets de recherche dont l'intérêt scientifique est depuis longtemps établi à l'aune des signes précédents. À leur instar, la diaspora bénéficie d'une telle considération depuis quelques années. Aujourd'hui, deux revues lui sont consacrées. La première, en anglais, est Diaspora: A Journal of Transnational Studies, publiée par la prestigieuse Oxford University Press et sponsorisée par le Zoryan Institute, un organisme à but non lucratif basé à Cambridge, Massachusetts et à Toronto, fondé par des chercheurs et acteurs de la société civile d'origine arménienne, et qui se "dédie à la recherche et à la documentation sur les questions relatives à l'histoire, à la politique, à la société et à la culture de l'Arménie et des Arméniens à travers le monde " (www.zoryan.org). La seconde, en français, est Diasporas: histoire et société, publiée à l'Université de Toulouse par le Centre interdisciplinaire de recherche et d'études sur les Juifs qui, tel que l'indique son nouveau nom, a intégré les diasporas. Outre ces deux revues spécialisées, plusieurs périodiques ont publié des numéros thématiques sur la diaspora. Ainsi, la Revue européenne des migrations internationales (Guillon et Ma Mung, 1992); le South Atlantic Quartely (Mudimbe et Engel, 1999) ; Issue (Brock, 1996) ; African Studies Review (Byfield, 2000) et L'Homme (Assayag et Bénei, 2000). 
21 L'état de la littérature sur la diaspora dissimule un retard des sciences sociales françaises à s'y intéresser, par comparaison aux anglo-américaines. Qui plus est, il inspire l'idée de l'incapacité des sciences sociales françaises à penser la diaspora noire plus spécifiquement. Je voudrais tenter, dans cette section, d'établir la validité de cette idée. Un corpus de trois séries de matériaux est analysé à cette fin. Ces matériaux sont sélectionnés pour leur pertinence au plan méthodologique. La première série consiste en la revue Diasporas : histoire et sociétés dont le centre d'intérêt est susceptible d'en faire un lieu approprié de réflexion sur la diaspora noire. Les périodiques spécialisés dans les études africaines constituent la deuxième. Cette aire de recherche étant la seule institutionnellement reconnue en France qui soit clairement dédiée à l'étude de populations d'ascendance africaine, ils peuvent logiquement servir d'espaces de discussion et de diffusion de travaux sur la diaspora noire. Enfin, la troisième série de matériaux a trait aux traductions françaises des recherches en langue étrangère sur la diaspora noire. L'adjonction de ces trois matériaux est souhaitable, car elle garantit une approche à la fois sectorielle et globale des sciences sociales françaises. Elle pourrait révéler, par exemple, que les revues que l'on soupçonne d'être plus réceptives à la recherche sur la diaspora noire, suivant nos propres présupposés ou la structuration des sciences sociales, ne le sont pas.

Des vingt-deux articles répartis en cinq numéros publiés à ce jour dans la revue française Diasporas : histoire et sociétés, trois articles, tous dans le cinquième numéro, s'intéressent à des composantes démographiques du monde noir. Ils sont de Daniel Friedman (2004), Anna Pondopoulo-Sanchez (2004) et Françoise Jourde-Raison (2004). Aucun ne prétend réfléchir sur le processus de construction d'une diaspora noire, ni n'use de ce concept en tant qu'outil d'analyse. Ils s'attellent plutôt à analyser la démarche de ces populations noires à s'investir d'une identité juive, dans les deux premiers cas, et hindouiste, dans le dernier. Les textes de Friedman et PondopouloSanchez qui, respectivement, rendent raison du processus sociohistorique de « communalisation» (Weber, $1995:$ 145sq) des Falacha d'Éthiopie et des Peul, avec la communauté diasporique juive en se définissant eux-mêmes comme des Juifs pourraient se ranger dans la littérature sur la diaspora juive. Tandis que celui de Raison-Jourde, qui porte sur l'en-définition de populations de Madagascar, est plus difficile à classer.

Parallèlement à Diasporas, les revues d'études africaines se prêtent aussi à la fonction de matériaux d'analyse. Parmi elles, deux, en particulier, ont été analysées: les Cahiers d'études africaines et Politique africaine. Elles sont toutes deux très représentatives du dynamisme ainsi que de la philosophie en vigueur dans le champ africaniste, et institutionnellement reconnues puisque sponsorisées et hébergées par des structures de recherche universitaires, qui sont respectivement l'EHESS et alternativement le Centre de Recherche d'Afrique Noire (CEAN) de l'Université de Bordeaux IV et le Centre de Recherche Africaine (CRA) de l'Université Paris I. Ces revues ont fait l'objet d'un dépouillement exhaustif sur la période 1994-2004, et d'un dépouillement aléatoire portant sur le premier numéro publié chaque année de 1960 à 1993 pour les Cahiers d'Études Africaines et de 1981 à 1993 pour Politique africaine. Le choix des années 1994-2004 se justifie au plan méthodologique, car il correspond à une période d'expansion significative des études sur la diaspora. La réflexion sur la diaspora noire en France était donc susceptible de se développer durant cet intervalle de temps. 

l'origine par les prestigieuses Harvard University Press et Verso, à Londres, il est paru aux éditions Kargo en France. On peut supposer que cette trajectoire éditoriale révèle une minoration de la diaspora noire dans la littérature française étant donné que cette maison d'édition, basée à Lille, n'est pas spécialisée dans les sciences sociales. Partant, l'entrée dans l'édition française par le truchement de Kargo signifie pour un ouvrage sociologique comme Black Atlantic un séjour - à court ou moyen terme - à la marge des sciences sociales, du moment que celles-ci constituent un champ de distribution de pouvoir et d'influence matériels ou symboliques qui sont davantage détenus, au plan de l'édition scientifique, par des maisons d'édition publiques telles les PUF, les éditions de l'EHESS, les éditions du CNRS ou des éditeurs privés dont Minuit, Fayard et Gallimard. manque d'intérêt des sciences sociales françaises pour la diaspora noire. Il importe à présent d'essayer d'en rendre raison. Je ne prétendrai pas restituer tous les facteurs qui contribuent à son explication. Je crois utile, en outre, de relever la difficulté qui est attachée à cet exercice en raison de mon positionnement vecteur de biais dont il importe que je prenne conscience dans ce cadre. Je suis à la fois professeur dans une université d'Amérique du Nord où le multiculturalisme est une politique officielle et la diaspora noire un projet achevé; noir et natif de l'Afrique jouissant de plusieurs citoyennetés; et enfin un produit de l'université française au sein de laquelle, malgré mon éloignement, je suis fortement intégré, davantage que dans bien d'autres universités, par mes réseaux de collaborations et mes intérêts de recherche. Ces 
identités et inscriptions pourraient me pousser à prendre la situation de la diaspora noire de l'Amérique du Nord pour un modèle universel, à généraliser ma condition d'acteur noir investi d'une identité diasporique à celle des populations noires de France ou à taire des attitudes ou dispositions caractéristiques de l'université française pour ne pas menacer les intérêts que je retire de mon intégration dans cette institution et ceux de mes collaborateurs. Ce n'est pas me soustraire à l'obligation de l'objectivation que de relever ces faits, mais au contraire m'en armer pour tendre à la démarche la plus scientifique possible, c'est-à-dire à l'effort de construction d'un savoir dans le souci d'assurer sa logique interne, d'établir la correspondance entre l'idée et les faits. Relever ces biais est aussi pour moi le moyen d'avouer la conception de la production scientifique dans laquelle je m'inscris et selon laquelle l'activité scientifique est un acte de création de connaissances contingentes, donc inscrites dans un contexte sociohistorique donné et élaborées à partir de présupposés qui participent de l'univers $\mathrm{du}$ chercheur, si bien que le discours scientifique s'avère aussi être moins un discours sur l'objet que sur la relation du chercheur avec celui-ci (Bourdieu, Wacquant, 1992). Sous l'angle de ce qui précède, une recherche postérieure à celle-ci est envisageable qui s'attellera à combler certains vides et démasquer quelques non-dits.

\section{Postcolonialisme/postmodernisme en France}

L'explication du déficit de recherche sur la diaspora noire gagnerait à prendre en compte la réception des théories postcoloniales et postmodernes dans les sciences sociales françaises. La raison en tient à la relation qu'entretient la réflexion sur la diaspora, lato sensu, à ces théories. En effet, de plus en plus d'auteurs s'accordent aujourd'hui à penser qu'une relation de dette lie la première aux secondes (Chivallon, 2004 ; Mbembe, 2005). Il est certes impossible de présenter exhaustivement toutes les données qui attestent cette dette, mais on peut réussir, par quelques exemples précis à en établir la preuve: ainsi, les manuels pédagogiques qui introduisent au postcolonialisme et postmodernisme accordent une attention significative à la production d'auteurs qui se trouvent être aussi parmi les plus marquants de la littérature sur la diaspora. Par exemple, l'anthologie consacrée par Greggory Castle (2001) aux études postcoloniales, par la place qu'il accorde à des contributeurs notables de la littérature sur la diaspora tels Hall, Radhakrishnan, Appiah, etc. témoigne du degré d'interpénétration entre elles. La même réflexion sur la diaspora est redevable à la contribution novatrice de penseurs influents du postmodernisme tels qu'Appadurai, Malkki et Gilroy. Le lien entre post-modernisme, postcolonialisme et la littérature sur la diaspora est tel que l'on est porté à se demander si la genèse de cette dernière est réellement dissociable du développement des précédents.

Relever ce lien, c'est avancer dans la compréhension du rapport des sciences sociales françaises à la diaspora, noire plus particulièrement. Postcolonialisme et postmodernisme influencent significativement l'évolution du débat scientifique et même celle de la pensée sociale dans le monde anglo-saxon, en particulier aux ÉtatsUnis, depuis les années 1980 (Castle, 2001; Young, 2001 ; Mbembe, 2005). Le marxiste américain, Russel Jacoby, pourfendeur du postcolonialisme, n'en reconnait pas moins sa forte influence dans le champ universitaire quand il écrit que: "La théorie postcoloniale occupe tout l'espace [de discussion]» (cité par Slemon, 2001: 100. Ma traduction). Mais, de manière surprenante, ces deux théories qui doivent en grande partie leur existence à la pensée de Foucault peinent à pénétrer les sciences sociales 
françaises. Elles s'y heurtent à une certaine circonspection : les chercheurs consacrés de France qui en revendiquent l'usage ou s'en réclament sont bien rares. L'article publié dans L'Homme par Jonathan Friedmann, sans être investi d'une quelconque représentativité, donne la mesure, par sa critique parfois sarcastique des travaux d'Appadurai ou de Malkki, de l'espèce de dédain dont le postmodernisme et le postcolonialisme sont frappés en France (Friedmann, 2000).

31 Pourtant l'émergence des théories postcoloniales et postmodernistes coïncide, en France, avec le moment d'une reconfiguration des sciences sociales qui leur offrait une opportunité de se positionner dans ce champ. De l'après-guerre jusqu'aux années 1980 environ, ce champ était construit sur une opposition tripolaire mettant en relation un courant marxiste, un courant durkheimien et un courant weberien, se prévalant tous du modernisme et rivalisant pour le monopole d'un certain positivisme, à savoir d'une idée selon laquelle la science est porteuse de vérité absolue et que par elle, les êtres humains peuvent accéder à une connaissance objective de la réalité (Kellner, 1999). La baisse d'influence du courant marxiste n'a pas entraîné la prédominance de l'un ni l'autre courants mais a libéré de l'espace que d'autres courants ont investi pour prendre part à la lutte de pouvoir qui se déroule dans les sciences sociales. Alors que cette recomposition était propice à une intégration du postcolonialisme et du postmodernisme dans les sciences sociales, il n'en a rien été. L'honnêteté exige de moi l'aveu d'une impuissance à éclairer cette faillite. Cependant, celle-ci n'a pu que retarder l'investissement - encore timide - par les sciences sociales françaises de la recherche sur la diaspora comme l'atteste la naissance de la revue Diasporas : histoire et société en 2002, donc un peu plus de 10 ans après la parution du premier numéro de son homologue américain : Diaspora: A Journal of Transnational Studies. La recherche sur la diaspora noire n'étant qu'une parcelle de la littérature sur la diaspora, lato sensu, sa condition en devient, par conséquent, tributaire.

32 Je résumerais ce qui précède en affirmant que la réflexion sur la diaspora noire est un pan de la littérature sur la diaspora, laquelle est portée par les théories postmodernistes ou postcoloniales. Or ces dernières aspirent encore à se positionner dans les sciences sociales françaises. Ce qui, par conséquent, se solde par l'absence de recherche sur la diaspora noire dans ce champ.

\section{Migration et non diaspora}

33 Nombre de passerelles ont été établies entre la migration et la diaspora dans les sciences sociales anglo-américaines en particulier. L'inventaire de cette littérature révèle que le concept de diaspora s'est largement prêté à l'analyse des dynamiques sociales des populations caractérisées par ailleurs d'immigrés au sens que Tribalat donne de ce terme, soit des individus nés dans un autre pays que celui de leur résidence (1995). Ni Safran (1991), ni Cohen (1997) ni Mudimbe et Engels (1999) n'ont manqué d'insister sur la relation entre migration et diaspora. Ce rapprochement n'est pas tout à fait arbitraire. Car, d'abord, les immigrés sont par définition des agents sociaux qui sont susceptibles d'intégrer dans leurs modes d'actions quotidiennes ainsi que dans leurs imaginaires le fait de leurs multiples inscriptions géographiques et culturelles, soit plus prosaïquement de leur existence en tant qu'individus intervenant parallèlement ou simultanément dans un univers national considéré comme le foyer d'origine et un autre qui est celui de leur adoption, de leur résidence. Ensuite, les immigrés sont souvent porteurs d'un projet, jamais réalisé dans quelque cas, de retour 
au foyer d'origine, qu'ils s'efforcent de présenter comme inéluctable par des entreprises symboliques telles l'acquisition d'un domicile au pays d'origine, la transmission de la langue maternelle aux enfants ; projet dont la sociologie française a démontré la signification à travers l'apparition quasi systématique et récurrente de sections ou chapitres consacrés au retour souvent dans les écrits des auteurs les plus importants de la migration. Or, les théoriciens classiques de la diaspora montrent bien que le retour comme projet entretenu dans un imaginaire ou démarche réelle à entreprendre est un critère important de définition de la diaspora : « un lien très fort au passé ou une résistance à l'assimilation, maintenant ou à l'avenir, sont nécessaires pour qu'émerge une conscience diasporique » écrit Cohen (1997 : 24. Ma traduction.)

On est logiquement autorisé à déduire de cette proximité entre migration et diaspora que l'importance de la première dans une littérature donnée crée un intérêt pour la seconde. Le cas des sciences sociales françaises infirme cependant cette corrélation. La section précédente a permis d'établir le très faible intérêt pour la diaspora dans ce champ, alors que la migration y jouit d'une solide institutionnalisation qui est attestée par la publication d'une revue académique (la REMI) et d'un périodique effectuant la jonction entre universitaires et « intervenants professionnels » (Hommes et Migrations), l'existence de plusieurs centres de recherche (MIGRINTER, URMIS etc.), de collections scientifiques (par exemple, CIEMI chez L'Harmattan) lui étant expressément dédiées, assorties de l'ouverture prochaine de la Cité de l'histoire de l'immigration.

Certainement, différents facteurs sont susceptibles d'expliquer l'absence de corrélation dans le contexte français. Les discours des acteurs directement impliqués, par exemple, mériteraient d'être analysés. Mais suivant la théorie du champ qui privilégie l'objectivation des structures par rapport à celle des individus, en raison de l'influence des structures sur les individus dont nombre de dispositions proviennent de celles-ci, mon analyse insistera davantage sur les structures.

En France, dans la littérature sur l'immigration, qui est la plus susceptible d'inclure la recherche sur la diaspora, a longtemps prévalu une approche que je qualifie de statocentrée adoptée souvent par les spécialistes dont De Wenden, Schnapper, Noiriel et Weil. Cette approche consiste à partir notamment de la construction institutionnelle de la migration, c'est-à-dire du discours et des archives produits par l'État pour étudier la migration. Par cette approche, les chercheurs s'exposent à un risque différent de celui qui menace leurs collègues adeptes de l'approche par le bas. C'est celui d'introduire la vision de l'État dans leur analyse même. Il ne s'agit pas d'un biais virtuel ainsi qu'en témoigne une série de faits. Un premier exemple: le concept d'assimilation qui a été central dans la littérature sociologique française sur la migration a été en même temps un terme quasi invariant du discours politique. L'ouvrage de Spire révèle que l'assimilation était pour l'État français à la fois un critère de sélection et un projet explicite à imposer ou en tout cas à expérimenter sur les étrangers (2005). Mieux, ce concept est tombé en désuétude dans la littérature au même moment qu'il a disparu du discours politique. Un deuxième fait est le discrédit qui pèse encore sur le concept de communauté dans les sciences sociales. Comme on le sait, il s'agit d'un concept qui renvoie à une réalité sociale que l'État français a toujours récusée conformément à son idéologie assimilationniste. Un certain déni s'ensuivit dans la littérature, alors que quelques travaux suggéraient qu'une attention soit portée à ce concept. Un dernier fait a trait à la discrimination comme concept ou objet d'étude. Depuis une trentaine d'années, ce concept est partie intégrante de la littérature anglo-américaine où il tient 
lieu d'outil d'analyse des rapports sociaux (Szymanski, 1976). Tandis qu'en France le numéro thématique publié en 1999 par la revue Hommes et Migrations est l'un des premiers à l'avoir introduit dans l'armature conceptuelle des sciences sociales (Dewitte, 1999). Or, Spire prouve, après d'autres, que jusque dans la sphère bureaucratique, la discrimination raciale a longtemps commandé les rapports sociaux non pas seulement entre nationaux et étrangers, mais entre citoyens français en France. Aux « Français musulmans d'Algérie ", par exemple, étaient refusés bien des avantages sociaux qui étaient parfois accordés même à des étrangers, mais d'ascendance européenne (2005: $129 \mathrm{sq}$ ). Le manque d'exploitation de ce concept par les chercheurs français coïncide avec le discours de l'État républicain qui affirme l'égalité de tous ses citoyens.

Les exemples précédents qui attestent l'influence du discours de l'État sur la recherche sur l'immigration aident à mieux comprendre la modeste présence de la recherche sur la diaspora dans les sciences sociales françaises. Dans leur texte introductif au numéro thématique de The Southern Atlantic Quaterly intitulé "Diaspora et Immigration", Mudimbe et Engel présentent la diaspora comme une sorte de nation au sein d'une nation (1999). En cela, la diaspora traduit l'existence d'un lien communautaire qui concurrence et peut éventuellement prévaloir sur le lien politique national. Suivant cette définition, la diaspora ne peut prétendre à une existence dans le discours de l'État français car, selon Schnapper, en France le lien politique est le lien social par excellence. Ce chercheur avance, du reste, une thèse intéressante : « [1]'idée même de la diaspora remet en question le principe de l'organisation politique moderne » (2001: 11). Reconnaître la diaspora dans le discours institutionnel si tant est que «dire, c'est faire » (Austin, 2002), reviendrait pour l'État français à avouer la fin même du pouvoir d'« assimilation » qu'il s'est auto-attribué et celui du monopole de l'allégeance politique de ses citoyens.

Je résumerais ce qui précède en soulignant que le rapport d'influence dans lequel s'inscrit le discours scientifique avec l'institutionnel (au sens d'étatique) en France peut amener les chercheurs à nier la validité du concept de diaspora. En tant que pan de la diaspora au sens large, la diaspora noire se trouve exposée aux effets de ce rapport d'influence, en plus de pâtir de la faible réception des théories postmodernes et postcoloniales - auxquelles elle est aussi associée - dans les sciences sociales. Il ne faudrait cependant pas en déduire que l'évolution de la réflexion sur la diaspora noire dans ce champ est entièrement fonction du développement de la littérature sur la diaspora en France. Je compte montrer dans la dernière section que les sciences sociales françaises fonctionnent pour le moment sur la base de présupposés et de dispositions structurelles qui entravent spécifiquement l'émergence d'une littérature substantielle sur les études sur la diaspora parce que, d'une part, elles récusent la catégorie de race et particulièrement le terme "noir » (Ndiaye, 2005), et d'autre part, parce qu'à travers l'anthropologie, elle repose sur une subdivision du savoir en aires géographiques.

\section{Le noir n'est pas, l'Afrique existe}

A - Par sa propre constitution sémantique, la diaspora noire renferme un obstacle qui réduit d'emblée son usage dans les sciences sociales françaises aujourd'hui. Car elle fait référence à la catégorie de race. Certes, comme le rappelle Mudimbe, dans la littérature ethnologique et anthropologique française $d u$ xVIII ${ }^{e}$ siècle et du début $d u x^{e}$ siècle, la 
catégorie de race était un outil d'analyse très prisé (1988: 13 sq). La race constituait, en effet, un outil explicatif des comportements, de l'état de développement technologique, voire de l'évolution et du statut socio-économiques d'un groupe, en même temps qu'elle déterminait sa place dans la chaîne de développement humain. Le fondateur de la Société d'Anthropologie de Paris, Paul Broca, fidèle au polygénisme dominant au XIx siècle, illustre bien cette tendance, lui qui écrivait en 1866 qu'aucun groupe humain « à la peau noire, aux cheveux laineux et au visage prognathe n'a pu s'élever spontanément jusqu'à la civilisation ». La représentativité de cette tendance se mesure à la réception très favorable dont elle avait bénéficié dans le milieu politique de la fin du XIX et du début $d u x^{e}$ ainsi que l'atteste l'adhésion d'hommes d'État tels Clemenceau à la thèse de l'inégalité des races. Du reste, Hervé Le Bras (1994) et Taguieff (1995) affirment que jusqu'après-guerre la catégorie raciale a persisté dans cette fonction d'outil d'analyse sociale et a même présidé à l'élaboration d'une politique d'immigration pour la France dans l'univers discret des bureaux et laboratoires de recherche. Le démographe Alfred Sauvy, actif dans l'appareil d'État gaulliste, en avait soutenu la promotion au rang de critère de sélection des immigrants, dans l'optique de la préservation de l'identité européenne de la France et de la «qualité biologique» de sa population, selon les termes de Taguieff (1995: 107).

Mais, une rupture s'est amorcée dans la littérature en sciences sociales vis-à-vis de cette tendance dominante. L'ouvrage de Lévi-Strauss, Race et histoire, constitue l'un des premiers où la validité de la catégorie de race est explicitement récusée (1987). L'ayant publié en 1952 à la demande de l'Unesco qui s'était attelée à la lutte contre le racisme, l'auteur substitue habilement, tout au long de son livre, culture à race. Se retranchant derrière l'idée que «la génétique moderne conteste » l'objectivité de la "notion purement biologique de race » (Lévi-Strauss, 1987 : 10). Lévi-Strauss, et, avec lui, une grande partie de la communauté des chercheurs, en a oublié de s'arrêter sur le processus social de conversion de cette caractéristique phénotypique en catégorie identitaire, et sur son pouvoir de structurer les relations entre les différents groupes sociaux tant dans l'espace national que dans l'espace international. La critique de la notion de race par les sciences sociales à partir des années 1950 a débouché sur son quasi-bannissement tant dans la littérature scientifique que dans le discours politique. Ainsi, le qualificatif de Noir a tout au plus été abandonné aux journalistes qui, à l'instar des rédacteurs du magazine Marianne, s'en servent pour composer des titres tels que La France noire. Du reste, et fait significatif, ce qualificatif est bien souvent délaissé dans le discours de ces derniers au profit de celui de Black - celui-ci serait-il plus moderne, véhiculerait-il moins un jugement de valeur? Pourtant, l'ouvrage de Poutignat et Streiff-Fénart (1995) suggère que les chercheurs de France ne sont pas plus convaincus que leurs collègues du monde anglo-américain qui en usent de l'absence de fondement objectif de la notion de race au plan " purement biologique».

41 Par leur rejet de la catégorie de race, en tant que construit social, les sciences sociales françaises s'interdisent de réfléchir sur la diaspora noire, puisque, comme l'a établi la première partie de cet article, l'identité diasporique au sein des populations noires de France est largement construite sur la mémoire collective de la traite négrière et la catégorie de race. Une telle attitude illustre la pertinence de l'idée de Bourdieu selon laquelle le champ scientifique fonctionne aussi sur «des catégories impensées de pensée qui délimitent les limites du pensable et prédéterminent la pensée » (Bourdieu et Wacquant, 1992 : 40. Ma traduction). 
sciences sociales sont surtout connues pour être un champ divisé en disciplines relativement autonomes les unes des autres (sociologie, économie, histoire, etc.). Elles connaissent aussi une division qui repose sur la distinction d'aires géographiques, depuis l'émergence de l'anthropologie à la suite de la géographie. La création de sociétés savantes au $\mathrm{XVIII}^{\mathrm{e}}$ siècle et de revues spécialisées a institutionnalisé cette division : l'Association Française des Anthropologues, la Société de Géographie œuvrent parallèlement à la Société des Océanistes, à la Société des Africanistes, bien que ce soient les mêmes chercheurs qui opèrent souvent dans les deux espaces; la revue Acta Geographica paraît à côté des Cahiers d'Études Africaines.

À travers les revues Cahiers d'Études Africaines, Politique africaine et le Journal des Africanistes, les « études africaines » ou « africanistes » jouissent d'une reconnaissance institutionnelle en France. Pour comprendre le déficit de réflexion sur la diaspora noire en France, je crois nécessaire d'analyser la structuration de l'africanisme français que je considère très représentatif $d u$ champ des sciences sociales pour les raisons suivantes. D'abord, il est un point d'articulation entre discipline et aire de spécialisation par le caractère interdisciplinaire de ses revues et de ses centres de recherches qui s'ouvrent à des chercheurs de toutes disciplines; il est ainsi ce que Dumont appelle une totalité partielle, c'est-à-dire une entité qui est englobée dans une autre en même temps qu'elle l'englobe. Ensuite, il est le seul espace institutionnellement constitué qui se consacre exclusivement aux dynamiques socioculturelles de personnes d'ascendance africaine. On ne recense pas en France un centre de recherche ni une revue exclusivement dédiés à l'étude des populations noires des Amériques.

44

À l'aune de ses revues et principaux centres de recherche (le Centre d'Études africaines, le Centre de Recherches Africaines, le Centre d'Études d'Afrique Noire), l'africanisme français est resté assez fidèle à sa mission originelle de production de savoirs sur les pratiques et expériences socioculturelles des populations socialisées et établies en Afrique. À l'époque de l'hégémonie de l'anthropologie, la définition de l'objet d'étude de l'africaniste était essentiellement basée sur le critère de l'ethnie présentée comme un groupe territorialement circonscrit. De nombreuses monographies ont ainsi été produites sur les Dogon (Dieterlen, par exemple), les Samo (Héritier), les Béti (Laburthe-Tolra), etc. Avec les Indépendances et l'urbanisation du continent, la définition de l'objet d'étude a connu un changement : le critère de la nationalité, donc le rapport à l'espace national de naissance et de socialisation, est devenu quasi dominant. La constitution de l'histoire en discipline autonome au sein de l'africanisme français, vers 1960, puis de la science politique ainsi que le souci des chefs des nouveaux États africains de promouvoir l'écriture d'une histoire nationale ont pesé d'un poids, qui reste à déterminer, sur l'évolution de l'objet d'étude africaniste. Toujours est-il que cet objet reste encore largement défini par le critère de la nationalité. La revue Politique africaine en fournit une illustration appropriée. Privilégiant la publication de numéros thématiques comme ligne éditoriale, elle se penche sur des questions telles que la démocratie, le multipartisme, la corruption, etc. Cependant, elle les aborde très souvent dans la perspective que Wimmer et Schiller désignent par «methodological nationalism» (2003), celle qui fait du territoire national l'espace par excellence d'observation et de compréhension des faits à analyser. Les titres de Politique africaine recèlent une forte référence à la catégorie nationale : « La Mauritanie : un tournant démocratique »; «Bénin»; «Le Cameroun dans l'entre- 
deux»; "L'Ouganda: une puissance régionale»; «Zimbabwe: l'alternance ou le chaos »; «RDC : la guerre vue d'en bas », etc.

Outre les revues, l'analyse des centres de recherche révèle des faits significatifs. Certains d'entre eux ont récemment intégré des chercheurs se spécialisant dans la diaspora noire ${ }^{2}$. Leur ouverture à des spécialistes de la diaspora noire n'a cependant pas eu d'effet tangible sur l'identité de ces centres au plan institutionnel. Par exemple, leurs noms sont restés inchangés. Or, le champ universitaire anglo-américain offre un excellent éclairage à ce propos. Aux États-Unis, par exemple, la vogue des cultural studies a été l'occasion de réaliser le rapprochement des études sur l'Afrique avec celles sur les peuples noirs des Amériques et d'ailleurs, par la création d'un département ou d'un centre de recherche conjoint souvent sous le nom de Black studies. La plupart des grandes universités s'y sont conformées. Temple University qui figure parmi les premières universités à s'engager dans cette voie (Small, 1999: 669) dispose d'un Africology Department, Harvard abrite désormais un Department of African and African American Studies et l'Université du Michigan un Center for Afro-American and African Studies. Dans la mouvance contraire, Columbia University fait figure d'exception en étant parmi les rares grandes universités à maintenir l'existence séparée d'un Institute of African American Studies et d'un Institute of African Studies. D'autres universités, à défaut d'un département, ont développé un programme d'enseignement et de recherche. Ainsi la New York University, qui, dans la présentation de son programme, insiste, en conformité avec l'idée dominante au sein de l'Université américaine, sur l'interpénétration des expériences historiques et culturelles des Noirs d'Afrique avec celles des Noirs des Amériques:

"The Africana Studies Program at New York University is devoted to the interdisciplinary and multi-continental study of modern black identities, culture, literature and politics. Africana Studies students explore the history and culture of black peoples in Africa, the Americas (including the Caribbean), and Europe, especially the emergence of black identities in the modern world. The program's two main areas of concentration are Pan-African History and Thought and Black Urban Studies. Pan-African History and Thought includes the study of such literary and political movements as the Harlem Renaissance, the Negritude movement, black consciousness, black feminism, and black intellectual leaders such as Aime Cesaire, Angela Davis, W.E.B. Du Bois, Zora Neale Hurston, C.L.R. James, Malcolm X, Kwame Nkrumah, and Leopold Senghor »

(http://www.nyu.edu/gsas/dept/ africana/).

La comparaison révèle la lenteur des études africaines en France à intégrer la réflexion sur la diaspora noire. La question se pose de savoir si les causes de cette lenteur relèvent de la superstructure ou de l'infrastructure. Le processus de redécoupage de l'objet des études africaines en entités géographiques plus réduites que l'Afrique aide à répondre à cette question. Ce redécoupage consacré par la notion d'Afrique au pluriel (les "Afriques») véhicule l'idée qu'il n'existe pas d'Afrique mais une diversité de territoires juxtaposés sur un même continent porteurs, chacun, de ses spécificités et autonomes vis-à-vis des dynamiques en cours sur le reste du continent. Ainsi distingue$\mathrm{t}$-on l'Afrique lusophone, l'Afrique anglophone, l'Afrique francophone ou pour d'autres chercheurs l'Afrique orientale, l'Afrique des Grands lacs, l'Afrique occidentale, l'Afrique australe. L'existence à l'Université de Pau et des pays de l'Adour d'un Centre de Recherche et d'Études sur les Pays d'Afrique Orientale ainsi que la publication par Karthala, un des principaux éditeurs d'ouvrages africanistes, de la revue Rupture 
spécialement consacrée à l'Afrique centrale semblent entériner ce redécoupage qui épouse presque la conception de ce continent à l'époque coloniale.

En résumé, le mode de construction de leur objet d'études réduit les possibilités des études africaines de concevoir la continuité ou au moins l'articulation entre les dynamiques des populations noires en Afrique et celles des Noirs établis hors du continent. Par conséquent, raisonner en termes de diaspora noire apparaît encore prématuré dans ce sous-champ.

\section{Conclusion}

Par comparaison aux sciences sociales anglo-américaines, les sciences sociales françaises ont tardivement encouragé la recherche sur la diaspora. Progressivement, celle-ci œuvre en faveur de son institutionnalisation avec l'existence d'une revue et d'un centre de recherche. Cependant, un déficit patent de réflexion sur la diaspora noire plus spécifiquement caractérise ce champ. Dans les lignes précédentes, quelques pistes ont été explorées pour l'explication de cette carence. Trois conclusions s'en sont dégagées : a) la réflexion sur la diaspora noire est un pan de la littérature sur la diaspora lato sensu qui n'a été intégrée que récemment dans les sciences sociales; b) cette réflexion est très associée aux théories postcoloniales ou postmodernistes qui restent marginalisées dans un champ scientifique français dont les acteurs souscrivent encore largement à la défense d'un universalisme considéré incompatible avec le respect de ces théories et de leurs avatars: Granjon (1994) et Lacorne (1994) l'ont d'ailleurs bien illustré qui établissent la manière très partiale dont le débat passionné sur le multiculturalisme aux États-Unis, dans les années 1990, avait été commenté par des penseurs français quasi unanimement acquis aux partisans de la préservation de la tradition universaliste; c) le déficit de réflexion sur la diaspora noire ressortit au bannissement de la catégorie de race dans les sciences sociales françaises : la réaction des chercheurs français au racisme scientifique ayant prévalu sous la III ${ }^{e}$ République a débouché, au-delà du rejet de l'idée de l'existence de la race en tant que catégorie biologique objective, sur sa négation comme construit social, et donc élément d'identification sociale présidant aux rapports sociaux entre groupes ou individus en société.

Au-delà de ces aspects, je crois utile de poursuivre la réflexion pour explorer les possibilités d'une relation entre le déficit de réflexion sur la diaspora noire et la présence numérique des Noirs au sein de l'Université française et dans l'espace public. La lecture de quelques travaux a suggéré cette piste. Slemon, par exemple, a montré que l'introduction des études postcoloniales en Grande-Bretagne est redevable à la création des Commonwealth studies qui a procédé de la mobilisation d'écrivains et chercheurs originaires de l'ancien Empire britannique en réaction à la politique des départements de littérature en langue anglaise de la Grande-Bretagne et de ses anciennes colonies de n'inscrire que des auteurs américains et britanniques au programme (2001 : 106). Ollivier, quant à elle, établit la corrélation entre, d'une part, l'accès progressif des femmes à des postes d'universitaires titulaires et leur présence croissante dans les programmes de maîtrise et de doctorat, et d'autre part, le renforcement des études féministes (2004). Souscrivant à l'idée de Gouldner selon laquelle «la théorie découle de la praxis des hommes dans leur entièreté et est informée par les vies qu'ils mènent » (cité dans Bourdieu et Wacquant, 1994: 38. Ma traduction), je crois 
pertinent, à des fins heuristiques au moins, de considérer le déficit de réflexion sur la diaspora noire dans les sciences sociales à la lumière de la qualité de la présence des Noirs dans les espaces universitaire et public français.

\section{BIBLIOGRAPHIE}

ASSAYAG Jackie et BÉNEI Véronique (coord.) (2000) « Intellectuels en diaspora et théories nomades ", L'Homme, 156, pp. 15-28.

AUSTIN John (2002) Quand dire, c'est faire, Paris, Seuil.

BARTH Fredrick (1995) «Les groupes ethniques et leurs frontières » in POUTIGNAT Philippe et STREIFF-FENART Jocelyne, Théories de l'ethnicité, Paris, P.U.F., pp. 203-249.

BLELARD Alain (1981) Négritude et politiques aux Antilles, Paris, Éditions caribéennes.

BORDES-BENAYOUN Chantal (2002) « Revisiter les diasporas », Diasporas, 1, pp. 11-21.

BOURDIEU Pierre et WACQUANT Loic (1992) An invitation to Reflexive Sociology, Chicago, Chicago U. P.

BOURDIEU Pierre (1991) « Le champ littéraire », Actes de la Recherche en Sciences Sociales, 89, pp. $4-46$

BOURDIEU Pierre (1984) Homo Academicus, Paris, Minuit.

BOURDIEU Pierre (1979) La distinction, Paris, Minuit.

BROCK, Lisa (coord.) (1996), « African Diaspora Studies », Issue, 24, 2.

BYFIELD Judith (coord.) (2000) « African Diaspora », African Studies review, 43, 1, pp. 1-9.

CASTLE Greggory (coord.) (2001) Postcolonial Discourses : an Anthology, London, Blackwell.

CHIVALLON Christine (2002) «La diaspora noire des Amériques : réflexions sur le modèle de l'hybridité de Paul Gilroy ", L'Homme, 161, pp. 51-74.

CHIVALLON Christine (2004) La diaspora noire des Amériques, Paris CNRS.

CLIFFORD James (1994) « Diasporas », Cultural Anthropology, 9, 3, pp. 302-338.

COHEN Robin (1997) Global Diasporas : An Introduction, UCL Press/University of Washington Press, London/Washington.

COLLECTIF (1983) Races imaginées et imaginaires, Paris, Maspéro/La découverte.

DEWITTE Philippe (coord.) (1999) « Connaître et combattre les discriminations », Hommes et Migrations, 1219.

DIOP Cheikh Anta (1954) Nations nègres et cultures, Paris, Présence africaine.

EDWARDS Brent H. (2003) The Practice of Diaspora : Literature, Translation and the Rise of Black Internationalism, Cambridge, Harvard U. P.

EDWARDS Brent H. (2001) « The Uses of Diaspora », Social Text, 19, 1, pp. 45-73. 
FOUCAULT Michel (2003) Les mots et les choses : une archéologie des sciences sociales, Paris, Gallimard « Tel».

FRIEDMAN Daniel (2004) « Mythes d'origine et généalogies rêvées des Falachas (Juifs d'Éthiopie) : de la généalogie individuelle à la généalogie groupale », Diasporas, 5, pp. 57-70.

FRIEDMAN Jonathan (2000) « Des racines et (dé) routes : tropes pour Trekkers », L'Homme, 156, pp.187-206.

GUILLON Michelle et MA MUNG Emmanuel (coord.) (1992), « La diaspora chinoise en Occident », Revue européenne des migrations internationales, 8, 3.

GILROY Paul (2003) L'atlantique noire: modernité et double conscience, Lille, Kargo.

GRANJON Marie-France (1994) «Le regard en biais : attitudes françaises et multiculturalisme américain (1990-1993) », Vingtième siècle, 43, pp. 18-28.

HALBWACHS Maurice (1968) La mémoire collective, Paris, P.U.F.

KASTORYANO Riva (1999) « Muslim Diaspora in Western Europe », The South Atlantic Quaterly, 98, 1/2, pp. 191-202.

KELLNER Douglas (1999) « Theorizing the Present Moment : Debates Between Modern and Postmodern Theory ", Theory and Society, 28, pp. 639-656.

KOSER Khalid (2002) «Une diaspora divisée : Transferts et transformations au sein de la diaspora érythréenne », Politique africaine, 85, pp. 64-74.

LACORNE Denis (1994) « Des coups de canon dans le vide ? "La civilisation occidentale" dans les universités américaines », Vingtième siècle, 43, pp. 4-17.

LE BRAS Hervé (1994) Le sang et le sol, La tour d'Aigues, éditions de l'Aube.

LÉVI-STRAUSS Claude (1987) Race et histoire, Paris, Denoël.

MARABLE Manning (1991) Race, Reform, and Rebellion, Jackson/London, University of Mississipi Press.

MASSING Andreas (2000) "The Wangara : An Old Soninke Diaspora in West Africa ", Cahiers d'études africaines, 158 (2), pp. 281-308.

MASSING Andreas (2004) "Baghayogho : A Soninke Muslim Diaspora in the Mande World ", Cahiers d'études africaines, 176, 4, pp. 887-922.

MBEMBE Achille (2005) "La République et l'impensé de la "race" ", in Pascal Blanchard et alii, La fracture coloniale, 139-153.

MUDIMBE Valentin et ENGEL Sabine (1999) «Introduction », The South Atlantic Quaterly, 98, 1/2, pp. 1-8.

NDIAYE Jean-Pierre (1962) Enquête sur les étudiants noirs, Paris, Réalités africaines.

NDIAYE Pap (2005) « Pour une histoire des populations noires en France : préalables théoriques », Le Mouvement social, 213, pp. 91-108.

OLLIVIER Michèle (2004) « Méthodologies féministes à l'ère de la mondialisation » Cahiers de recherche du GREMF, 88, pp. 45-70.

PATTERSON Tiffany et KELLY Robin (2000) « Unfinished Migrations : Reflections on the African Diaspora and the Making of the Modern World », African Studies Review, 43,1, pp. 11-45. 
PONDOPOULO-SANCHEZ Anna (2004) « Comment les Peuls sont-ils devenus des Juifs? Au sujet de l'une des versions de l'origine orientale des Fulbé (Afrique de l'Ouest, début du Xx siècle », Diasporas, 5, pp. 87-96.

POUTIGNAT Philippe et STREIFF-FENART Jocelyne (1995) Théories de l'ethnicité, Paris, P.U.F. RAISON-JOURDE Françoise (2004) « À la recherche d'ancêtres hindo-bouddhistes pour Madagascar : une généalogie rêvée en situation coloniale (année 1920-1950) », Diasporas, 5 , pp. 98-108.

SAFRAN William (1991) «Diasporas in Modern Societies : Myth of Homeland and Return », Diaspora, 1, pp. 83-99.

SCHNAPPER Dominique (2001) « De l'État-nation au monde transnational : du sens et de l'utilité du concept de diaspora », Revue Européenne des Migrations Internationales, 17, 2, pp. 9-36.

SCHNAPPER Dominique (1991) La France de l'intégration, Paris, Gallimard.

SLEMON Stephen (2001) « Postcolonial Critical Theories », in Greggory Castle, Postcolonial Discourse, London, Blackwell, pp. 100-115.

SMALL Mario « Departmental Conditions and the Emergence of New Disciplines: Two cases in the Legitimation of African-American Studies », Theory and Society, 28, pp. 659-707.

SPIRE Antoine (2005) Étrangers à la carte, Paris, Grasset.

SZYMANSKI Albert (1976) « Racial Discrimination and White Gain », American Sociological Review, 41, 403-414.

SUNDQUIST Eric www.hup.harvard.edu/reviews/GILBLA R. html (consulté le 3-09-2005).

TAGUIEFF Pierre-André (1995) « Face à l'immigration : mixophobie, xénophobie ou sélection », Vingtième siècle, 1995, pp. 103-131.

TEVOEDJRE, Albert (1956) « Un scandale terrifiant ! », L'Étudiant d'Afrique Noire, juin-septembre, pp. 1-2.

TRIBALAT Michèle (1995) Faire France : une grande enquête sur les immigrés et leurs enfants, Paris, La Découverte.

WEBB James (1995) « The Evolution of the Idaw al-Hajj Commercial Diaspora », Cahiers d'études africaines, 138/139, 2/3, pp. 455-475.

WEBER Max (1995) Économie et société, tome 2, Paris, Plon « Pocket ».

WEIL Patrick (1995) « Racisme et discrimination dans la politique française de l'immigration : 1938-1945/1974-1995 », Vingtième siècle, 47, 77-102.

WIMMERS Andreas et Schiller Nina (2003) « Methodological Nationalism, the Social Science, and the Study of Migration : An Essay in Historical Epistemology », International Migration Review, 37, 3, pp. 576-610.

YOUNG Robert (2001) «Colonialism and the Desiring Machine » in Greggory Castle, Postcolonial Discourse, London Blackwell, pp. 74-97.

ZONGO Mahamadou (2003) «La diaspora burkinabé en Cote d'Ivoire », Politique africaine, 90, pp. 113-126. 


\section{NOTES}

1. Mes remerciements aux lecteurs anonymes ainsi qu'à Gervais Carpin pour leurs excellentes suggestions. Warm thanks to Huzzefa S. Rashid for his assistance.

2. Ainsi le Centre d'Études d'Afrique Noire qui, depuis peu, compte parmi ses membres, Christine Chivallon, l'une des rares spécialistes de la diaspora noire en France.

\section{RÉSUMÉS}

$\mathrm{Au}$ cours des quinze dernières années, la réflexion sur la diaspora noire a connu un développement important dans le milieu universitaire anglo-américain. En revanche, en France, un déficit patent caractérise les sciences sociales à ce sujet. Pourtant les chercheurs français ne manquent pas de raison de s'intéresser à la diaspora noire. La France a constitué depuis l'entredeux-guerres un espace majeur d'élaboration d'une identité diasporique noire. Du fait de lois ségrégationnistes en place aux États-Unis, de l'occupation américaine de Haïti entre 1915 et 1939 et de l'absence d'un enseignement supérieur et parfois secondaire dans ses colonies d'Afrique, la France a été la destination de milliers de jeunes Noirs, dont une part significative d'intellectuels, d'écrivains et d'étudiants. Transcendant les différences ethniques, nationales et linguistiques entre ses membres, cette élite s'était attelée à l'objectif ambitieux de coordination de tous les Noirs de la terre, dans l'objectif de l'émancipation de la race noire et de l'Afrique. Cet article s'interroge sur les fondements épistémologiques du déficit de réflexion sur la diaspora noire dans les sciences sociales françaises.

In the last fifteen years, the reflection on the African Diaspora has been granted a very significant place in the Anglo-American academia. In contrast, in France, the social sciences have appeared uninterested in this literature. Yet, French researchers have many reasons to take part in this reflection. Since the interwar period, France has been a major site for activists working towards the construction of a Black diasporic identity. Because of segregationist laws in the US, the American occupation of Haiti, and the lack of (post-) secondary education in its African colonies, France has been the destination of thousands of young Blacks among whom a significant proportion of intellectuals, writers and students. Moving beyond its ethnic, national and linguistic differences among its members, these elite took on the ambitious objective of coordinating Blacks all around the world for the emancipation of Africa and the black race. This article questions the epistemological foundations of the dearth of reflection on Black diaspora in the French social sciences.

Durante los últimos quince años, la reflexión sobre la diáspora negra ha conocido un desarrollo fulgurante en los círculos universitarios anglo-americanos. En contraste, en Francia, un déficit patente sobre este tema caracteriza a las ciencias sociales. Sin embargo no faltan razones para que los investigadores franceses se interesen al tema. Desde el periodo entre las dos guerras Francia fue un gran espacio de elaboración de une identidad de la diáspora negra. La atracción de los Estados Unidos estaba limitada por la existencia de leyes segregacionistas y la ocupación norteamericana en Haití entre 1915 y 1939. Falta de establecimientos de enseñanza superior y a veces mismo secundaria en sus colonias africanas, Francia fue la destinación de muchos de jóvenes negros, una parte significativa de ellos fueron intelectuales, escritores y estudiantes. Transcendiendo las diferencias étnicas, nacionales y lingüísticas entre sus miembros, esta elite 
tenía el objetivo ambicioso de coordinar a todos los negros de la tierra, con el objetivo de emancipar a la raza negra y a África. Este artículo cuestiona los fundamentos epistemológicos del déficit de reflexión sobre la diáspora negra en las ciencias sociales francesas.

INDEX

Mots-clés : diaspora, Africains

Index géographique : Afrique

\section{AUTEUR}

\section{ABDOULAYE GUEYE}

Professeur, University of Ottawa, Department of Sociology, 550 Cumberland, Ottawa, Ont., K1N 6N5, Canada. 